\title{
Development Of New Damage Tolerant Alloys For Age-Forming
}

\author{
M.J. Starink, I. Sinclair, N. Gao, N. Kamp, P.J. Gregson, P.D. Pitcher ${ }^{1}$ \\ A. Levers ${ }^{2}$ and S. Gardiner ${ }^{2}$ \\ Materials Research Group, School of Engineering Sciences, University of Southampton, \\ Southampton, SO17 1BJ \\ ${ }^{1}$ QinetiQ, Farnborough GU14 OLX, UK \\ ${ }^{2}$ Airbus UK, Filton, Bristol BS99 7AR, UK
}

Keywords: fatigue resistance, 2xxx alloys, DSC, creep forming, toughness.

\begin{abstract}
The suitability of age forming for the shaping of damage tolerant structures is investigated by formulating and testing new alloy-age forming combinations. The alloy formulation process is driven initially by modelling of strength and semi-quantitative understanding of other microstructure-property relations. Using this a range of $\mathrm{Al}-\mathrm{Cu}-\mathrm{Mg}-\mathrm{Li}-(\mathrm{Zr}-\mathrm{Mn})$ based alloys predicted to provide yield strengths in aged condition comparable with incumbent 2024-T351 alloy for lower wing skins are selected. It is shown that several of these new alloys after artificial aging representative of age-forming have proof strength (PS), fatigue crack growth resistance (FCGR) and toughness that are comparable or better than 2024-T351. UTS to PS ratios of the new alloys are lower than 2024-T351.
\end{abstract}

\section{Introduction}

Age forming has substantial potential cost benefits for the production of curved aluminium structures. In age forming (also known as creep forming or creep age forming) a curved component is formed using a combination of pressure and temperature typically in the range of those used for ageing of heat treatable alloys $\left(150-190^{\circ} \mathrm{C}\right)$. The technique is currently applied to production of upper wing skins of commercial aircraft, but as incumbent lower wing skin alloys lose their damage tolerant properties upon ageing, it is not currently applied for lower wing skins. The present multicentre collaborative project created within the Defence and Aerospace Research Partnership for Advanced Metallic Airframes (DARP-AMA) is aimed at investigating the applicability of age forming for the forming of damage tolerant structures by formulating and testing new alloy-age forming combinations. These newly formulated alloys are designed to meet requirements for ageformability (incl. springback) and mechanical properties, including strength and damage tolerance.

The alloy development project is backed up by extensive analysis of the microstructure, the corrosion resistance and the key mechanical properties of the alloys, using techniques such as 3 dimensional atom probe (3DAP), scanning and transmission electron microscopy (SEM, TEM) and differential scanning calorimetry (DSC) [1]. In the present contribution we will present the compositions and test results of a range of alloys and we will discuss the general prospects for application of age forming to produce damage tolerant medium strength curved aluminium structures.

\section{Alloy selection and property modelling.}

The alloy selection process aims to define alloys with the following properties:

A In the artificially aged/age formed condition, key properties are to be similar to 2024T351. This concerns specifically yield strength, fatigue crack growth (FCG) resistance, toughness, ultimate tensile strength and corrosion resistance.

B Good age formability. Age formability will be identified via creep tests at 150 to $190^{\circ} \mathrm{C}$. 
Some of the requirements are conflicting, for instance a high yield strength can be deleterious to damage tolerance (fatigue \& toughness).

The strategy for alloy selection has been based on available processing-microstructure-property models and general metallurgical knowledge. To date a total of 11 alloys have been designed and made. The compositions are given in Table 1 . Alloy 5 is essentially 2024, and is used as a baseline reference (comparisons are then made between materials from the same small-scale casting route). The following sections give a brief outline of the considerations and models that were used in designing the alloys.

Yield Strength

For a new age formable alloy to replace 2024-T351 a the yield strength equal to or better than that of 2024-T351 is required. Increasing yield strength is detrimental to toughness [2] and is also thought to reduce age formability, and hence the aim for the new alloy is to have a yield strength that does not markedly exceed the minimum requirement. Thus alloys were designed such that the expected strength after artificial ageing is about $325 \mathrm{MPa}$ (equal to 2024-T351). As a complete model is not yet available at this stage of the work, simplified models based only on strengthening due to S phase precipitation and solution strengthening (see e.g. [3]) are currently employed. This simplified model has proved reasonably successful with alloys achieving the strength target with an accuracy of about $25 \mathrm{MPa}$. For all alloys, compositions can be further fine-tuned to obtain specific strength level.

Fatigue Crack Growth Resistance

For the new age-formable alloys we require that the fatigue crack growth resistance is similar to 2024-T351. It is thought that fatigue resistance is especially enhanced by the occurrence of crack closure $[4,5]$, which in turn is enhanced by the presence of shearable precipitates and zones. The presence of coarse intermetallics is thought to be detrimental for fatigue resistance (at higher stress intensity levels at least), and increases in strength may also have a detrimental effect on fatigue resistance (note however that when strength is increased through the addition of more shearable particles, crack closure effects may also be significantly enhanced, as is commonly thought to be the case for Al-Li alloys). Grain structure and dispersoid content will also influence fatigue resistance. Toughness

Substantial Li content leading to $\delta^{\prime}\left(\mathrm{Al}_{3} \mathrm{Li}\right), \delta$ and $\mathrm{T}_{2}$ will have a negative influence on toughness, particularly in terms of in-service embrittlement due to $\delta^{\prime}$ formation $[6,7,8]$. Hence Li contents are limited to levels that do not give rise to $\delta$ '. As indicated above, increased strength may be expected to have a negative impact on toughness, and hence alloys are designed to have strength levels that do not significantly exceed minimum requirements.

Age formability / Creep

As creep is a thermally activated process, the types of shapes to be attained during age forming can always be achieved simply by increasing the temperature of the process. However, increasing the temperature will generally be detrimental to the mechanical property balance, especially the FCGR.

In general creep is reduced when stable particles are present in the alloy, and this is for instance used in the creep resistant $\mathrm{Al}$ 2618. Non-shearable strengthening precipitates are thought to influence creep. Of the three main precipitates that occur in artificially aged Al-Cu-Mg based alloys, $\mathrm{S}^{\prime}, \mathrm{T}_{1}$ and $\Omega, \mathrm{T}_{1}$ is thought to provide the greatest resistance against creep, whilst $\mathrm{S}^{\prime}$ is thought to provide the least resistance against creep [9]. (This may be the reason for the enhanced creep resistance of Al-Cu-Mg-Ag type alloys in which $\Omega$ formation is stimulated by the presence of Ag. $\Omega$ precipitates coarsen very slowly for $\mathrm{T}<200^{\circ} \mathrm{C}$, and this may be an important reason for the reduction in creep they provide.) Thus, for the present alloys, formation of $T_{1}$ and $\Omega$ is considered to be undesirable, and Ag additions are avoided.

Microalloying 
To select alloys that may be suitable for age forming, a number of strategies and alloy compositions have been considered. Generally, to provide for shearable particles in an age formed alloy with an aim of achieving good FCGR, it would be preferable to keep the alloy in underaged condition. One way of achieving this is by slowing down the precipitation process through the addition of suitable (micro-) alloying elements. In general, addition of alloying elements with a high vacancy binding energy will cause a reduction in the amount of free vacancies that are available for the formation of vacancy loops, which are important nucleation sites for S'. In principle a wide range of elements can reduce the free vacancy concentration, and for the present work $\mathrm{Li}$ is selected because of its combined beneficial influence on FCGR and reduced ageing rates.

In considering the above, and with the aid of simplified strength modelling compositions in the range Al-(1.5-2.5)Cu-(0.8-1.2)Mg-(0.5-1.5)Li are selected. Having presented the simplified models and considerations that were used to design the alloys, the analysis of the properties of the alloys, which will ultimately decide whether alloys are suitable for age forming, is presented in the next sections. The results will provide feedback to the modelling effort, and this will be used in the subsequent cycles of alloy design/refinement.

\section{Experimental Procedures}

At the present stage of the work 11 alloys have been manufactured at QinetiQ, Farnborough. Compositions of selected alloys are presented in Table 1. Casting and subsequent processing included: casting, stress relieve at $300^{\circ} \mathrm{C} / 24 \mathrm{hrs}$, homogenizing by heating at $10^{\circ} \mathrm{C} / \mathrm{h}$ from 400 to $485^{\circ} \mathrm{C}$ or $495^{\circ} \mathrm{C}$ (temperature selection based on DSC analysis of incipient melting), hold for 24 hours, upset forge, hot rolling to $20 \mathrm{~mm}$ plate at $\sim 420^{\circ} \mathrm{C}$, solution heat treatment (Alloy 1 at $525^{\circ} \mathrm{C}$, Alloys 2, 5 and 6 at $495^{\circ} \mathrm{C}$, and Alloy 3 and 4 at $513^{\circ} \mathrm{C}$ ), cold water quench, and stretch by $2.5 \%$. Ageing treatments for 6 up to 48 hrs at 130, 150 and $190^{\circ} \mathrm{C}$ were conducted. For comparison also standard 2024-T351 commercially produced plate material was tested.

Vickers hardness values were obtained from surfaces ground with \#1200 grade SiC-paper. Before testing, the test machine was calibrated using a standard test block. Four indentations were made on each specimen with a $20 \mathrm{~kg}$ load and a mean is reported.

Tensile tests were carried out according to ASTM standard E-8. Specimens were taken in the longitudinal (L) orientation (i.e. parallel to the rolling direction).

In the first instance only ranking of fracture toughness is required for the present work, rather than exact $\mathrm{K}_{\mathrm{IC}}$ measurements. As such, Charpy slow bend testing has been used in this work (following ASTM standard E-812-91). A 'crack strength' $\left(\sigma_{c}\right)$ is obtained from the Charpy slow bend tests and is defined as the maximum value of the nominal (net-section) stress that a cracked specimen is capable of sustaining. It may

\begin{tabular}{cccccc}
\hline Alloy & $\mathrm{Cu}$ & $\mathrm{Mg}$ & $\mathrm{Mn}$ & $\mathrm{Zr}$ & $\mathrm{Li}$ \\
\hline 1 & 2.72 & 0.45 & 0.43 & 0.004 & - \\
2 & 2.81 & 1,05 & 0.41 & 0.002 & - \\
3 & 2.27 & 1.03 & 0.01 & 0.106 & 1.56 \\
4 & 2.24 & 0.94 & 0.42 & 0.001 & 1.60 \\
5 & 4.30 & 1.46 & 0.43 & 0.061 & 0.17 \\
6 & 4.34 & 1.37 & 0.42 & 0.001 & - \\
\hline
\end{tabular}

Table 1: Compositions of the alloys studied. shown

to be sensitive to changes in the plane-strain fracture toughness, with the normalised crack strength $\sigma_{\mathrm{c}} / \sigma_{\mathrm{y}}$ being correlated with $\mathrm{K}_{\mathrm{IC}} / \sigma_{\mathrm{y}}$. Specimens were taken in longitudinal-transverse (LT) orientation. Fatigue crack propagation tests were carried out according to ASTM standard E647 using small compact tension samples ( $\mathrm{W}=24 \mathrm{~mm}, \mathrm{~B}=10 \mathrm{~mm}$ ), using a loading frequency of $40 \mathrm{~Hz}$ and R-ratios of 0.1 and 0.4. The LT loading orientation was again used. All samples (Charpy slow bend and fatigue tests) were extracted from the mid-thickness of the plates. For optical microscopy samples were ground and subsequently polished using 3 and 1 micron DP-Spray, and OP-S 
Suspension. Keller's reagent (2ml $\mathrm{HF}$ (48\%), $3 \mathrm{ml} \mathrm{HCl,} 5 \mathrm{ml} \mathrm{HNO}_{3}, 190 \mathrm{ml} \mathrm{H} \mathrm{H}_{2}$ ) was used to reveal the microstructure of the polished materials. Details of DSC and TEM experiments are presented elsewhere [1,3].

\section{Results and Analysis}

The microstructure and microstructure development of the alloys was studied using DSC, TEM and 3DAP. Details of this work are presented elsewhere, and at present it is noted all alloys aged at $150^{\circ} \mathrm{C}$ contained mostly clusters and all alloys aged at $190^{\circ} \mathrm{C}$ contained predominantly $\mathrm{S}$ phase precipitates. Alloy 1 contained some $\Omega$ phase. None of the aged alloys contained appreciable $\delta$ ', $\delta$ or $\mathrm{T}_{2}$.

The ultimate tensile stress (UTS) and $0.2 \%$ proof stress (PS) for selected alloys aged at 150 and $190^{\circ} \mathrm{C}$ are shown in Fig. 1. On the basis of strength the alloys can be divided in three groups: the highest strengths are found for alloy 5 (2024), the lowest strengths are found for alloy 1 and medium strengths are found for alloys 2, 3, 4 and 6. It can be seen from Fig. 1 that strength increases very slowly at $150^{\circ} \mathrm{C}$; whilst at $190^{\circ} \mathrm{C}$ strength increases much quicker reaching a peak within 10 to $30 \mathrm{~h}$. Alloys 3, 4 and 6 have almost identical UTS both aged at 150 and $190^{\circ} \mathrm{C}$ although they have distinct yield strength, which is quite different from other alloys.
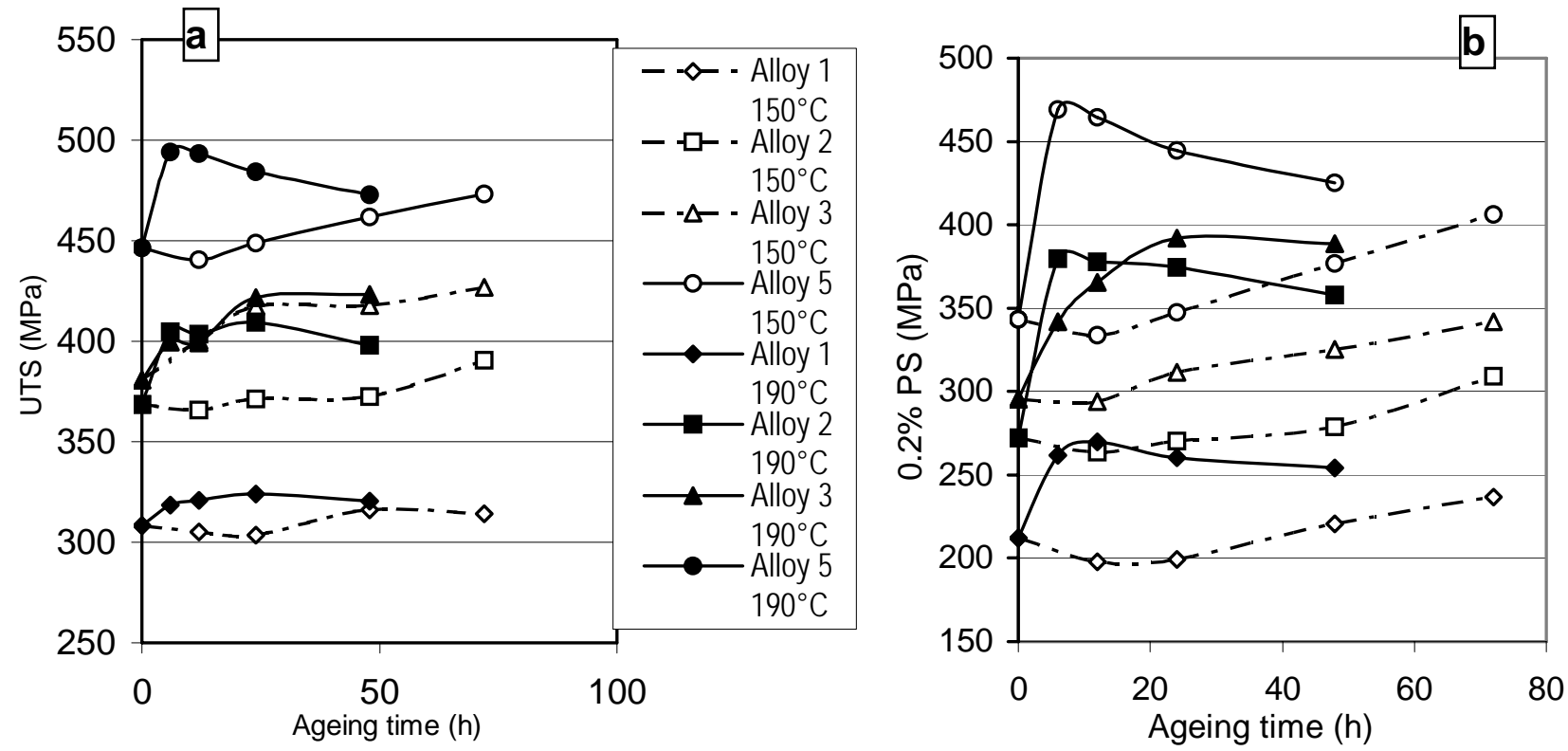

Fig. 1: UTS (a) and PS (b) for alloys 1,2,3,5 aged at 150 and $190^{\circ} \mathrm{C}$

In view of the objectives of the alloy design process the yield strengths obtained after $190^{\circ} \mathrm{C}$ ageing are quite encouraging for alloys 2, 3, 4, and 6. Yield strength increases up to $40 \%$, from 270 $\mathrm{MPa}$ in solution treatment condition to $380 \mathrm{MPa}$ for alloy 2 in $190^{\circ} \mathrm{C} / 6 \mathrm{hrs}$, which is higher than 2024-T351 standard. For these alloys the values of yield strength at $190^{\circ} \mathrm{C}$ are close to the values of tensile strength (shown in Fig. 7), which means that this alloy in the artificially aged condition possesses a low $\sigma_{U T S} / \sigma_{y}$ ratio. The latter is thought to be a largely unavoidable consequence of ageing / age forming resulting from the strengthening contribution by precipitates (e.g. S) which are sheared on extensive yielding. Shearing on yielding will cause a reduction is precipitate strengthening thus causing a low $\sigma_{\mathrm{UTS}} / \sigma_{\mathrm{y}}$ ratio. Ageing at $150^{\circ} \mathrm{C}$ for alloys 3,4 and 6 does not provide the required yield strength until $48 \mathrm{~h}$, but ageing at $190^{\circ} \mathrm{C}$ quickly improves the yield strength to exceed the 2024 standard. The advantage for these alloys over other alloys is that the yield strength keeps high even until 48 hrs, which means that it is still not overaged at this stage. As 
expected, Alloy 5 is very strong when aged at $190^{\circ} \mathrm{C}$, and may be considered too strong for age forming.

It was found that the strength-hardness relationship is not always straightforward. Data for alloys 1 and 2 plotted in Fig. 2 shows a very good linear relationship between hardness and UTS, which

gives $\mathrm{HV} \cong 3.07 \sigma_{\text {UTS }}$. However, the yield strength is not a single simple linear function of the hardness. Our data, when plotted, follows two lines. One is for $\mathrm{HV} \cong 4.2 \sigma_{0.2}$ shown as the bottom line and another is a line close to ultimate tensile strength, with a relationship $\mathrm{HV} \cong 3.3 \sigma_{0.2}$. These high values correspond to data for alloy 2 aged at $190^{\circ} \mathrm{C}$. In Fig.3, crack strengths (a measure of the toughness) for alloys 2-6 and 2024-T351 are plotted vs. their proof strengths. This data shows

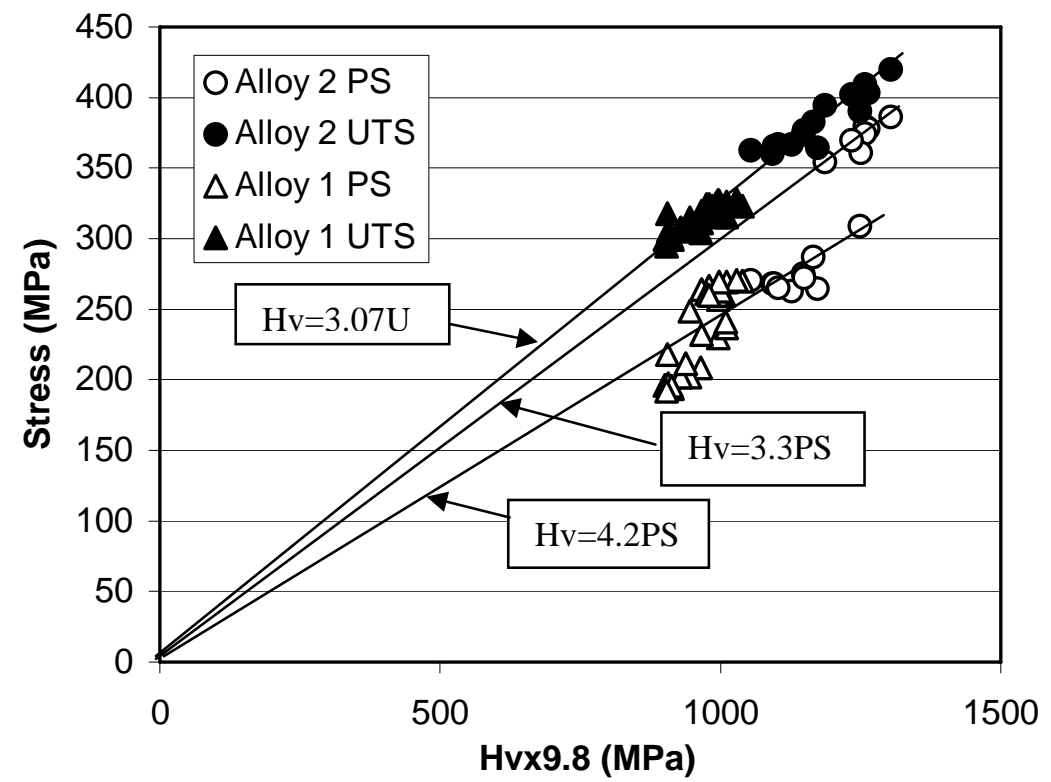

Fig. 2: Graph of hardness vs proof stress for alloys 1 and 2. that alloys 2, 3, 4 and 6 have

crack strengths that are comparable or better than 2024-T351, whilst the artificially aged 2024 (alloy 5) has crack strengths that are less than 2024-T351. These results are consistent with the principles of toughness-strength and toughness-microstructure relations as outlined above. Particularly, these results confirm that 2024 alloys loose toughness when given an artificial ageing treatment because the strength increases, whilst the newly designed alloys 2, 3 and 6 have good toughness in artificially aged condition, because strength is comparable to 2024-T351 and $\delta^{\prime}\left(\mathrm{Al}_{3} \mathrm{Li}\right), \delta$ and $\mathrm{T}_{2}$ are avoided. This indicates that provided the alloy design rules outlined above are used, age formed alloys with toughness-strength combinations comparable or better than 2024-T351 can be designed.

In Fig. 4 the FCG rates of alloys 2-6 and 2024-T351 are plotted as a function of $\Delta K$ for $R=0.1$. These results show that FCG resistance of the new alloys is for most of the $\Delta \mathrm{K}$ are comparable or better that the incumbent 2024-T351. Especially the Mn and Li containing alloy 4 shows a very strongly improved FCG resistance. As yet incomplete results for $\mathrm{R}=0.4$ show similar trends.

Fig. 3: Graph of crack strength (from Charpy slow bend tests) vs proof stress for alloys 2-6 and 2024-T351.

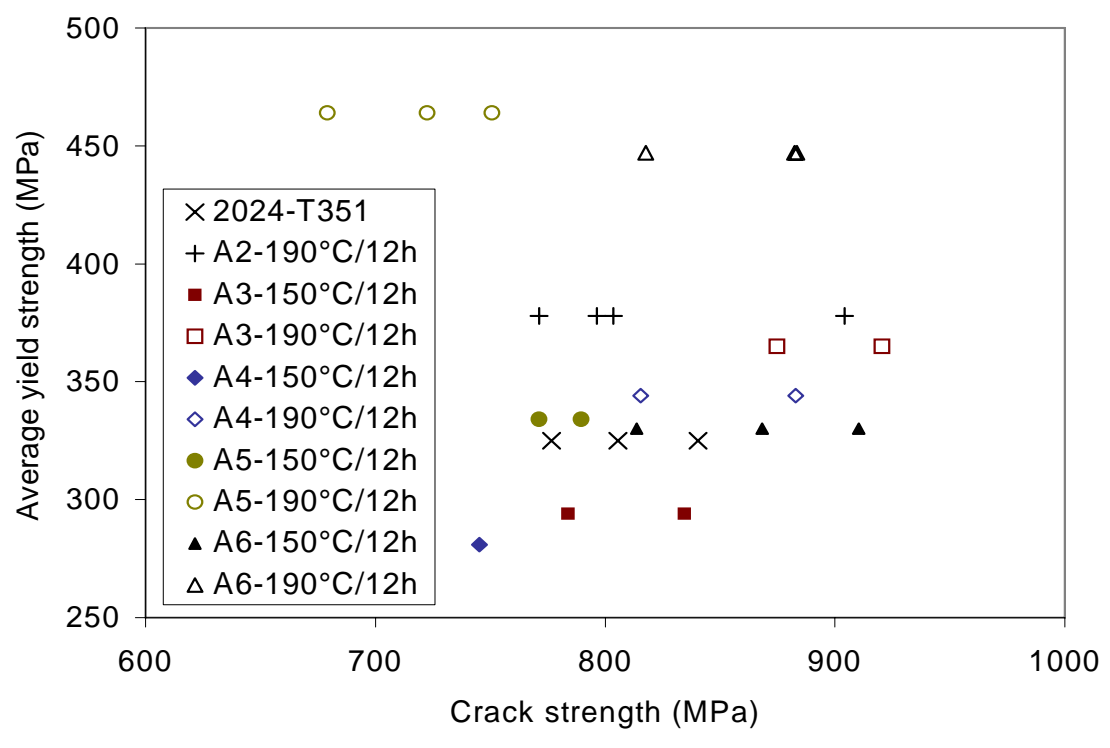




\section{Concluding Remarks}

The present results show that after artificial aging representative of age-forming several of the newly designed alloys have PS, FCG resistance and toughness that are comparable or better than incumbent 2024-T351, but UTS to PS ratios of the new alloys are lower than incumbent 2024-T351. These results suggest that there is a potential for using the new alloys in damage tolerant age-formed curved structures (e.g. lower wing skin) provided that the reduced UTS to PS ratio can be accommodated within the design. The latter may be achieved by using the improved PS, FCG resistances and toughness properties of the some of the new alloys.

Fig. 4: Fatigue crack growth rate vs $\Delta \mathrm{K}$ for $\mathrm{R}=0.1$.

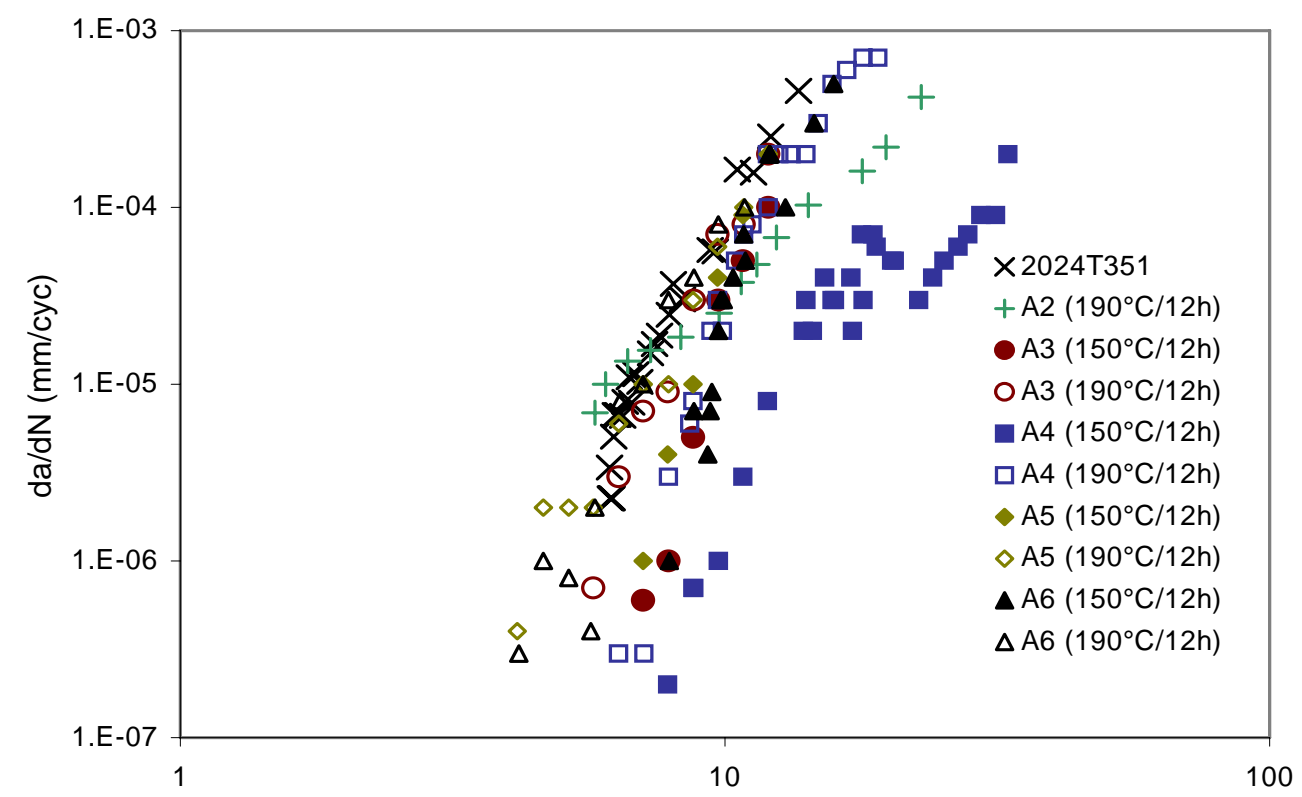

\section{Acknowledgements}

The authors would like to acknowledge the financial support from EPSRC, QinetiQ (formerly DERA), MOD and Airbus UK. Alcoa Europe (formerly British Aluminium) is acknowledged for funding the production of 2 alloys and for support in the conception stage of the project. J. Haddock, C.J. Peel and K.A.Q. O’Reilly are acknowledged for support in the conception stage of the project. Dr M. Joyce is acknowledged for performing additional materials testing.

\section{References}

1 N. Gao, L. Davin, S. Wang, A. Cerezo and M.J. Starink, Mater Sci Forum, Vol. 396-402 (2002) 923-28

2 N. Kamp, I. Sinclair and M.J. Starink, Metall. Mater. Trans. Vol 33A (2002) 1125-1136

3 M.J. Starink, P. Wang, I. Sinclair and P.J. Gregson, Acta Mater. Vol 47 (1999) 3855-3868

4 Y. Xu, P.J. Gregson and I. Sinclair, Mater. Sci. Foum Vol. 331-3 (2000) 1525-30

5 M.R. Parry, S. Syngellakis and I. Sinclair, Mater. Sci. Forum Vol 331-3 (2000) 1473-78

6 M.J. Starink, A.J. Hobson, I. Sinclair and P.J. Gregson, Mater. Sci. Eng. Vol 289A (2000) 130-42

7 P.J. Gregson and H.F. Flower, Acta Metall. Mater. Vol. 33 (1985) 527-37

8 P.J. Gregson and S.A. Court, Scr. Metall. Mater. Vol. 30 (1994) 1359-63

9 Taminger, K.M.B. et al. Proc. ICAA-6, Toyohasi, Japan, 1998 